\title{
Symmetry and Nonexistence of Positive Solutions for a Fractional Laplacion System with Coupled Terms
}

Rong Zhang ( $\nabla$ rongzhangnnu@163.com )

Nanjing Normal University

\section{Research Article}

Keywords: Fractional Laplacian, Moving planes, Radially symmetric, Doubling Lemma, Singularity estimates

Posted Date: January 10th, 2022

DOI: https://doi.org/10.21203/rs.3.rs-1024420/v1

License: (c) (1) This work is licensed under a Creative Commons Attribution 4.0 International License.

Read Full License 


\title{
Symmetry and Nonexistence of Positive Solutions for a fractional Laplacion System with coupled terms
}

\author{
RONG ZHANG \\ Institute of Mathematics \\ School of Mathematical Sciences \\ Nanjing Normal University \\ Nanjing, 210023, China \\ rongzhangnnu@163.com
}

\begin{abstract}
In this paper, we study the problem for a nonlinear elliptic system involving fractional Laplacion:

$$
\left\{\begin{aligned}
(-\Delta)^{\frac{\alpha}{2}} u & =|x|^{\gamma} u^{p} v^{q+1}, \\
(-\Delta)^{\frac{\beta}{2}} v & =|x|^{\tau} u^{p+1} v^{q},
\end{aligned}\right.
$$

where $0<\alpha, \beta<2, p, q>0$ and $\max \{p, q\} \geq 1, \alpha+\gamma>0, \beta+\tau>0, n \geq 2$. First of all, while in the subcritical case, i.e. $n+\alpha+\gamma-p(n-\alpha)-(q+1)(n-\beta)>0$, $n+\beta+\tau-(p+1)(n-\alpha)-q(n-\beta)>0$, we prove the nonexistence of positive solution for the above system in $\mathbb{R}^{n}$. Moreover, though Doubling Lemma to obtain the singularity estimates of the positive solution on bounded domain $\Omega$. In addition, while in the critical case, i.e. $n+\alpha+\gamma-p(n-\alpha)-(q+1)(n-\beta)=0, n+\beta+\tau-(p+1)(n-\alpha)-q(n-\beta)=0$, we show that the positive solution of above system are radical symmetric and decreasing about some point by using the method of Moving planes in $\mathbb{R}^{n}$.
\end{abstract}

Mathematics Subject Classification (2020): 35R11, 35A10, 35B06.

Key words: Fractional Laplacian; Moving planes; Radially symmetric; Doubling Lemma; Singularity estimates.

\section{Introduction}

In this paper, we study the following system

$$
\left\{\begin{array}{l}
(-\Delta)^{\frac{\alpha}{2}} u=|x|^{\gamma} u^{p} v^{q+1}, \\
(-\Delta)^{\frac{\beta}{2}} v=|x|^{\tau} u^{p+1} v^{q},
\end{array}\right.
$$

where $0<\alpha, \beta<2, p, q>0$ and $\max \{p, q\} \geq 1, \alpha+\gamma>0, \beta+\tau>0, n \geq 2$.

We also assume that

$$
n+\alpha+\gamma-p(n-\alpha)-(q+1)(n-\beta) \geq 0 ;
$$




$$
n+\beta+\tau-(p+1)(n-\alpha)-q(n-\beta) \geq 0 ;
$$

The system (1.1) and the corresponding parabolic problem appear in the study of static Schrödinger theory and Bose Einstein condensate with two components ([15]). It also can be used to describe competition of biological population. In recent years, the fractional Laplacian has attracted much attention from the mathematical community due to its nonlocality and widespread applications. It can be used to model diverse physical phenomena. For instance, in the diffusion process, the operator was used to derive heat kernel estimates for many symmetric jump-type processes (see [1]) and to study the acoustic wave equation. In astrophysics, it is used to model the dynamics in the Hamiltonian chaos (see [25]). It also has various applications in probability and finance, in which this operator is defined as the generator of -stable Lvy processes that represent random motions, such as the Brownian motion and the Poisson process (see [8]), anomalous diffusion and quasi-geostrophic flows, turbulence and water waves, molecular dynamics, and relativistic quantum mechanics of stars, see ([3], [5], [24]) and it also plays an important roles in the theory of nonlinear partial differential equations.

The fractional Laplacian in $\mathbb{R}^{n}$ is a nonlocal pseudo-differential operator, we assuming the form

$$
\begin{aligned}
(-\Delta)^{\frac{\alpha}{2}} u & =C_{n, \alpha} P V \int_{\mathbb{R}^{n}} \frac{u(x)-u(y)}{|x-y|^{n+\alpha}} d y \\
& =C_{n, \alpha} \lim _{\varepsilon \rightarrow 0} \int_{\mathbb{R}^{n} \backslash B_{\varepsilon}(0)} \frac{u(x)-u(y)}{|x-y|^{n+\alpha}} d y .
\end{aligned}
$$

where $C_{n, \alpha}$ is a normalization constant and $P V$ represents the Cauchy principal value.

\subsection{Nonexistence and Symmetry}

In this subsection, we study the following system

$$
\left\{\begin{array}{cc}
(-\Delta)^{\frac{\alpha}{2}} u=|x|^{\gamma} u^{p} v^{q+1}, & x \in \mathbb{R}^{n}, \\
(-\Delta)^{\frac{\beta}{2}} v=|x|^{\tau} u^{p+1} v^{q}, & x \in \mathbb{R}^{n}, \\
u \geq 0, v \geq 0, & x \in \mathbb{R}^{n} .
\end{array}\right.
$$

where $0<\alpha, \beta<2, p, q>0$ and $\max \{p, q\} \geq 1, \alpha+\gamma>0, \beta+\tau>0, n \geq 2$.

We define the solution of (1.3) in the distribution sense. Let

$$
\mathcal{L}_{\alpha}=\left\{u: \mathbb{R}^{n} \rightarrow \mathbb{R} \mid \int_{\mathbb{R}^{n}} \frac{|u(x)|}{1+|x|^{n+\alpha}} d y<\infty\right\},
$$

and

$$
\mathcal{L}_{\beta}=\left\{u: \mathbb{R}^{n} \rightarrow \mathbb{R} \mid \int_{\mathbb{R}^{n}} \frac{|u(x)|}{1+|x|^{n+\beta}} d y<\infty\right\},
$$

Obviously, the integral in (1.3) is well defined for $u \in \mathcal{L}_{\alpha} \cap C_{\text {loc }}^{1,1}, v \in \mathcal{L}_{\beta} \cap C_{\text {loc }}^{1,1}$.

It is not easy to deal with the problems involving a nonlocal fractional Laplacian operator due to the nonlocal charactrristic of these operators. The first method to handle such problems is known as the extended method, which was introduced 
by Caffarelli and Silvestre [4]. Namely, a nonlocal problem ivolving the fractioal Laplacian is transformed into a higher dimensional local prolem.

The study of the solution $u: \mathbb{R}^{n} \rightarrow \mathbb{R}$ of the equation involving fractional Laplacian needs its extension $U: \mathbb{R}^{n} \times[0, \infty) \rightarrow \mathbb{R}$ given by

$$
\left\{\begin{array}{l}
\operatorname{div}\left(y^{1-\alpha} \nabla \mathcal{U}\right)=0, \quad(x, y) \in \mathbb{R}^{n} \times[0, \infty) \\
\mathcal{U}(x, 0)=g(x)
\end{array}\right.
$$

Then

$$
(-\Delta)^{\frac{\beta}{2}} g(x)=-C_{n, \beta} \lim _{y \rightarrow 0^{+}} y^{1-\beta} \frac{\partial \mathcal{U}}{\partial y}, \quad x \in \mathbb{R}^{n} .
$$

The other method is the integral equations method, such as method of moving planes in integral forms to study their equivalent corresponding integrals $([16],[17])$. That is, if we choose the integral equations method to study the well-known nonlinear partical differential equation:

$$
(-\Delta)^{\frac{\alpha}{2}} u=u^{\frac{n+p}{n-p}}
$$

we need an equaivalent integral form:

$$
u(x)=\int_{\mathbb{R}^{n}} \frac{u^{\frac{n+p}{n-p}}(y)}{|x-y|^{n-\alpha}} d y .
$$

Recently, Zhang et al. in [28] studied the problem for a nonlinear elliptic system involving fractional Laplacion:

$$
\left\{\begin{aligned}
(-\Delta)^{\frac{\alpha}{2}} u & =|x|^{\tau} u^{p} v^{q+1} \\
(-\Delta)^{\frac{\alpha}{2}} v & =|x|^{\tau} u^{p+1} v^{q}
\end{aligned}\right.
$$

where $0<\alpha<2, p, q>0$ and $\max \{p, q\} \geq 1, \tau \geq 0, n \geq 2$. They showed that the positive solution of above system are radially symmetric and decreasing about origin by using the method of Moving planes in $\mathbb{R}^{n}$. Moreover, while in the subcritical case $p+q+1<\frac{n+\alpha+2 \tau}{n-\alpha}$, they proved the nonexistence of positive solution for the above system in $\mathbb{R}^{n}$, then though Doubling Lemma to obtained the singularity estimates of the positive solution on bounded domain $\tilde{\Omega}$.

After routine calculations like in [12], assume $u, v \in \mathcal{L}_{\alpha} \cap C_{l o c}^{1,1}$ and satisfy

$$
\begin{aligned}
\int_{\mathbb{R}^{n}}(-\Delta)^{\frac{\alpha}{4}} u(x)(-\Delta)^{\frac{\alpha}{4}} \phi(x) d x & =\int_{\mathbb{R}^{n}}|x|^{\tau} u^{p}(x) v^{q+1}(x) \phi(x) d x, \\
\int_{\mathbb{R}^{n}}(-\Delta)^{\frac{\alpha}{4}} v(x)(-\Delta)^{\frac{\alpha}{4}} \phi(x) d x & =\int_{\mathbb{R}^{n}}|x|^{\tau} u^{p+1}(x) v^{q}(x) \phi(x) d x,
\end{aligned}
$$

for any $\phi(x) \in C_{0}^{\infty}$, where

$$
\int_{\mathbb{R}^{n}}(-\Delta)^{\frac{\alpha}{4}} u(x)(-\Delta)^{\frac{\alpha}{4}} \phi(x) d x
$$

is defined by the Fourier transform

$$
\int_{\mathbb{R}^{n}}|\xi|^{\alpha} \hat{u}(\xi) \overline{\hat{\phi}(\xi)} d \xi
$$


where $\hat{u}$ and $\hat{\phi}$ are the Fourier transform of $u$ and $\phi$ respectively. Zhang et al. also obtained that system (1.3) as defined above is equivalent to integral system

$$
\left\{\begin{array}{l}
u(x)=\int_{\mathbb{R}^{n}} \frac{|y|^{\tau} u^{p}(y) v^{q+1}(y)}{|x-y|^{n-\alpha}} d y, u>0 \text { in } \mathbb{R}^{n}, \\
v(x)=\int_{\mathbb{R}^{n}} \frac{|y|^{\tau} u^{p+1}(y) v^{q}(y)}{|x-y|^{n-\alpha}} d y, v>0 \text { in } \mathbb{R}^{n} .
\end{array}\right.
$$

Ma et al. in [19] studied the nonexistence of positive solutions for the following fractional Hénon system

$$
\left\{\begin{aligned}
(-\Delta)^{\frac{\alpha}{2}} u=|x|^{a} v^{p}, & x \in \mathbb{R}^{n}, \\
(-\Delta)^{\frac{\alpha}{2}} v=|x|^{b} u^{q}, & x \in \mathbb{R}^{n}, \\
u \geq 0, v \geq 0, & x \in \mathbb{R}^{n},
\end{aligned}\right.
$$

where $0<\alpha<2,1 \leq p, q<\infty, a, b \geq 0, n \geq 2$. Using a direct method of moving planes, they have been proved the non-existence of positive solution in the subcritical case $1<p<\frac{n+\alpha+a}{n-\alpha}, 1<q<\frac{n+\alpha+b}{n-\alpha}$. Moreover, they also proved (1.8) equivalence the following integral system

$$
\left\{\begin{array}{l}
u(x)=C_{1} \int_{\mathbb{R}^{n}} \frac{|y|^{a} v^{p}(y)}{|x-y|^{n-\alpha}} d y, \\
v(x)=C_{2} \int_{\mathbb{R}^{n}} \frac{|y|^{b} u^{q}(y)}{|x-y|^{n-\alpha}} d y,
\end{array}\right.
$$

under certain suitable conditions.

In [14], Li concerned the following elliptic system

$$
\left\{\begin{aligned}
-\Delta u & =(q+1) u^{q} v^{p+1}, & & u>0 \in \mathbb{R}^{n}, \\
-\Delta v & =(p+1) u^{q+1} v^{p}, & & v>0 \in \mathbb{R}^{n},
\end{aligned}\right.
$$

where $n \geq 3, p, q>0$ and $\max \{p, q\} \geq 1$. They discussed the nonexistence of positive solution in subcritical case and stable solution in supercritical case, the necessary and sufficient conditions of classification in the critical case, and by using the Liouville theorem of (1.10), they estimated boundary blow-up rate, i.e. $u(x), v(x) \leq \operatorname{Cdist}^{-\frac{2}{p+q}}(x, \partial \Omega)$, where $\Omega \subset \mathbb{R}^{n}$ is a bounded domain.

Chen [6] proved that when $n \geq 3$ and $\min \{p, q\}>0$, if $(u, v)$ solve (1.10), then it also solves the integral system

$$
\left\{\begin{array}{l}
u(x)=C_{1} \int_{\mathbb{R}^{n}} \frac{|y|^{a} u^{q}(y) v^{p+1}(y)}{|x-y|^{n-2}} d y \\
v(x)=C_{2} \int_{\mathbb{R}^{n}} \frac{|y|^{b} u^{q+1}(y) v^{p}(y)}{|x-y|^{n-2}} d y
\end{array}\right.
$$

for some positive constants $C_{1}, C_{2}$.

In [2], the author dealed with the local and global behaviour of the positive solution of the semilinear elliptic system in $\mathbb{R}^{n}(n \geq 3)$

$$
\begin{cases}-\Delta u=|x|^{\sigma} u^{q} v^{p+1}, & u>0 \in \mathbb{R}^{n}, \\ -\Delta v=|x|^{\sigma} u^{q+1} v^{p}, & v>0 \in \mathbb{R}^{n},\end{cases}
$$

where $\sigma, p, q \in R$, and $p, q>0$. Their main results are the fact that the solution satisfy Harnack inequality when $p+q+1<\frac{n+2}{n-2}$ in the local estimates. If not, they also given the precise behaviour of the solution. 
Li et al. in [12] studied the following weighted system of partial differential equations

$$
\left\{\begin{aligned}
(-\Delta)^{\frac{\alpha}{2}} u=|x|^{-s} v^{p}, & x \in \mathbb{R}^{n}, \\
(-\Delta)^{\frac{\alpha}{2}} v=|x|^{-t} u^{q}, & x \in \mathbb{R}^{n}, \\
u \geq 0, v \geq 0, & x \in \mathbb{R}^{n},
\end{aligned}\right.
$$

where $p, q>1,0<\alpha<n$ and $0 \leq s, t<\alpha$. They first established the equivalence between partial differential system and weighted integral system

$$
\left\{\begin{array}{l}
u(x)=\int_{\mathbb{R}^{n}} \frac{v^{p}(y)}{|x-y|^{n-\alpha}|y|^{s}} d y, \\
v(x)=\int_{\mathbb{R}^{n}} \frac{u^{q}(y)}{|x-y|^{n-\alpha}|y|^{t}} d y .
\end{array}\right.
$$

Then, in the critical case of $\frac{n-s}{q+1}+\frac{n-t}{p+1}=n-\alpha$, they showed that every pair of positive solution $(u, v)$ are radially symmetric about the origin. While in the subcritical case, they proved the nonexistence of positive solution.

Remark 1. Inspired by aforementioned work, we can obtain that system (1.3) as defined above is equivalent to integral system

$$
\left\{\begin{array}{l}
u(x)=\int_{\mathbb{R}^{n}} \frac{|y|^{\gamma} u^{p}(y) v^{q+1}(y)}{|x-y|^{n-\alpha}} d y, u>0 \text { in } \mathbb{R}^{n}, \\
v(x)=\int_{\mathbb{R}^{n}} \frac{|y|^{\tau} u^{p+1}(y) v^{q}(y)}{|x-y|^{n-\beta}} d y, v>0 \text { in } \mathbb{R}^{n} .
\end{array}\right.
$$

The following is our main theorems.

If $p$ and $q$ is subcritical, we will show that there actually is no positive solution.

Theorem 1.1. Assume that $u \in \mathcal{L}_{\alpha} \cap C_{l o c}^{1,1}, v \in \mathcal{L}_{\beta} \cap C_{\text {loc }}^{1,1}$, and $\max \{p, q\} \geq 1$. Let $(u, v)$ is a pair of positive solutions for (1.3), then in subcritical case, namely, $n+\alpha+\gamma-p(n-\alpha)-(q+1)(n-\beta)>0, n+\beta+\tau-(p+1)(n-\alpha)-q(n-\beta)>0$, (1.3) has no positive solutions.

If $p$ and $q$ is critical, we will show that the solution is radical symmetric.

Theorem 1.2. Assume that $u \in \mathcal{L}_{\alpha} \cap C_{l o c}^{1,1}, v \in \mathcal{L}_{\beta} \cap C_{l o c}^{1,1}$, and $\max \{p, q\} \geq 1$. Let $(u, v)$ is a pair of positive solutions for (1.3), then in critical case, namely, $n+\alpha+\gamma-p(n-\alpha)-(q+1)(n-\beta)=0, n+\beta+\tau-(p+1)(n-\alpha)-q(n-\beta)=0$, (1.3), $u$ and $v$ must be radially symmetric with the some center.

\subsection{Singularity estimate}

Finally, we consider a problem on the bounded domain

$$
\begin{cases}(-\Delta)^{\frac{\alpha}{2}} u=|x|^{\gamma} u^{p} v^{q+1}, & x \in \Omega, \\ (-\Delta)^{\frac{\beta}{2}} v=|x|^{\tau} u^{p+1} v^{q}, & x \in \Omega,\end{cases}
$$

where $0<\alpha, \beta<2, p, q>0$ and $\max \{p, q\} \geq 1, \alpha+\gamma>0, \beta+\tau>0, n \geq 2$, $\Omega \subset \mathbb{R}^{n}$ is a bounded domain. We will estimate boundary blow-up rate. The following Doubling Lemma which is developed by Polacik-Quittner-Souplet [20] plays a key role to obtain the estimate.

Lemma 1.3 (Doubling Lemma) Let $(X, d)$ be a complete metric space and let $\varnothing \neq$ $D \subset \Sigma \subset X$, with $\Sigma$ closed. Set $\Gamma=\Sigma / D$. Finally let $M: D \rightarrow(0, \infty)$ be 
bounded on compact subsets of $D$ and fix a real $K>0$. If $y \in D$ is such that $M(y) \operatorname{dist}(y, \Gamma)>2 K$, then there exists $x \in D$ such that

$$
\begin{gathered}
M(x) \operatorname{dist}(x, \Gamma)>2 K, M(x) \geq M(y), \\
M(z) \leq 2 M(x) \text { for all } z \in D \cap \overline{B_{K / M(x)}(x)} .
\end{gathered}
$$

Based on this Lemma, we establish the equivalence between the Liouville theorem of (1.3) and the estimate of boundary blow-up rate for solutions of (1.16), and combining with the nonexistence of the positive solution of (1.3) we can obtain that the following result.

Theorem 1.4. Let $n+\alpha+\gamma-p(n-\alpha)-(q+1)(n-\beta)>0, n+\beta+\tau-(p+$ $1)(n-\alpha)-q(n-\beta)>0$. The following conclusions are equivalent:

(i) The system (1.3) has no bounded positive classical solution;

(ii) Positive solution $(u, v)$ of (1.16) satisfies estimates of the boundary blow-up rate

$$
\begin{aligned}
& u(x) \leq \operatorname{Cdist}^{-\frac{\alpha}{p+q}}(x, \partial \Omega), \\
& v(x) \leq \operatorname{Cdist}^{-\frac{\beta}{p+q}}(x, \partial \Omega) .
\end{aligned}
$$

Thoughout the paper, we use $C$ to denote a generic constant whose value may be different from line to line or even in the same line.

The paper is organized as follows: In Section 2, we given some notations and some necessary lemma. In Section 3, we complete the proof of Theorem 1.1 and Theorem 1.2 by the moving plane. In Section 4, we use the Double Lemma to prove Theorem 1.4.

\section{Preliminaries}

In this section, we will given some notations and some necessary lemma to proof of Theorem 1.1 and Theorem 1.2.

\section{$2.1 \quad$ Notations}

In this subsection, we given some notatons, in order to study the symmetry and monotonicity of positive solutions for fractional systems (1.3) by a direct method of moving planes. We are not able to carry the method of moving planes on $u$ and $v$ directly since there is no any decay conditions on $u$ and $v$.

To overcome this difficulty, we make a Kelvin transform. Denote

$$
\left\{\begin{array}{l}
\bar{u}(x)=\frac{1}{\left|x-z^{0}\right|^{n-\alpha}} u\left(\frac{x-z^{0}}{\left|x-z^{0}\right|^{2}}+z^{0}\right), \\
\bar{v}(x)=\frac{1}{\left|x-z^{0}\right|^{n-\beta}} v\left(\frac{x-z^{0}}{\left|x-z^{0}\right|^{2}}+z^{0}\right),
\end{array}\right.
$$

be the Kelvin transform centered at any given point $z^{0}$.

When $z^{0}$ is the origin, while the proof for a general $z^{0}$ is entirely similar. Namely, (2.1) becomes

$$
\left\{\begin{array}{l}
\bar{u}(x)=\frac{1}{|x|^{n-\alpha}} u\left(\frac{x}{|x|^{2}}\right), \\
\bar{v}(x)=\frac{1}{|x|^{n-\beta}} v\left(\frac{x}{|x|^{2}}\right)
\end{array}\right.
$$


be the Kelvin transform of $u$ and $v$ centered at the origin.

It is easy to see

$$
\bar{u}(x) \sim \frac{1}{|x|^{n-\alpha}}, \bar{v}(x) \sim \frac{1}{|x|^{n-\beta}}, \quad \text { for large }|x| .
$$

By (2.2), we have

$$
\begin{aligned}
(-\Delta)^{\frac{\alpha}{2}} \bar{u}(x) & =\frac{1}{|x|^{n+\alpha}}(-\Delta)^{\frac{\alpha}{2}} u\left(\frac{x}{|x|^{2}}\right) \\
& =\frac{1}{|x|^{n+\alpha+\gamma}} u^{p}\left(\frac{x}{|x|^{2}}\right) v^{q+1}\left(\frac{x}{|x|^{2}}\right) \\
& =\frac{1}{|x|^{n+\alpha+\gamma-p(n-\alpha)-(q+1)(n-\beta)}} \bar{u}^{p}(x) \bar{v}^{q+1}(x) .
\end{aligned}
$$

Similarly, we have

$$
(-\Delta)^{\frac{\beta}{2}} \bar{v}(x)=\frac{1}{|x|^{n+\beta+\tau-(p+1)(n-\alpha)-q(n-\beta)}} \bar{u}^{p+1}(x) \bar{v}^{q}(x) .
$$

Let $a=n+\alpha+\gamma-p(n-\alpha)-(q+1)(n-\beta), b=n+\beta+\tau-(p+1)(n-\alpha)-q(n-\beta)$, then (1.3) becomes

$$
\left\{\begin{array}{cc}
(-\Delta)^{\frac{\alpha}{2}} \bar{u}=\frac{1}{|x|^{a}} \bar{u}^{p} \bar{v}^{q+1}, & x \in \mathbb{R}^{n}, \\
(-\Delta)^{\frac{\beta}{2}} \bar{v}=\frac{1}{|x|^{b}} \bar{u}^{p+1} \bar{v}^{q}, & x \in \mathbb{R}^{n}, \\
\bar{u} \geq 0, \quad \bar{v} \geq 0, & x \in \mathbb{R}^{n} .
\end{array}\right.
$$

Define the moving planes

$$
T_{\lambda}=\left\{x \in \mathbb{R}^{n} \mid x_{1}=\lambda, \lambda \in \mathbb{R}\right\},
$$

and the region to the left of the plane

$$
\Sigma_{\lambda}=\left\{x \in \mathbb{R}^{n} \mid x_{1}<\lambda, \lambda \in \mathbb{R}\right\} .
$$

Let

$$
x^{\lambda}=\left(2 \lambda-x_{1}, x_{2}, \cdots, x_{n}\right)
$$

be the reflection of the point $x=\left(x_{1}, \cdots, x_{n}\right)$ about the plane $T_{\lambda}$, and

$$
u_{\lambda}(x)=u\left(x^{\lambda}\right), v_{\lambda}(x)=v\left(x^{\lambda}\right) .
$$

Now, assume that $(\bar{u}, \bar{v})$ solves the fractional system (1.3). We denote

$$
\left\{\begin{array}{l}
U_{\lambda}(x)=\bar{u}_{\lambda}(x)-\bar{u}(x), \\
V_{\lambda}(x)=\bar{v}_{\lambda}(x)-\bar{v}(x),
\end{array}\right.
$$

to compare the value of $\bar{u}_{\lambda}(x)$ with $\bar{u}(x)$ and $\bar{v}_{\lambda}(x)$ with $\bar{v}(x)$ respectively.

By system (1.3), we have

$$
\left\{\begin{array}{l}
(-\Delta)^{\frac{\alpha}{2}} U_{\lambda}(x)=\frac{1}{\left|x^{\lambda}\right|^{a}} \bar{u}_{\lambda}^{p}(x) \bar{v}_{\lambda}^{q+1}(x)-\frac{1}{|x|^{a}} \bar{u}^{p}(x) \bar{v}^{q+1}(x), \\
(-\Delta)^{\frac{\beta}{2}} V_{\lambda}(x)=\frac{1}{\left|x^{\lambda}\right|^{b}} \bar{u}_{\lambda}^{p+1}(x) \bar{v}_{\lambda}^{q}(x)-\frac{1}{|x|^{b}} \bar{u}^{p+1}(x) \bar{v}^{q}(x) .
\end{array}\right.
$$




\subsection{Necessary Lemma}

In order to proof of Theorem 1.1 and Theorem 1.2, we will show the key ingredients in the method of moving planes such as narrow region principle and decay at infinity.

Lemma 2.1 (Narrow Region Principle [27]) Let $\Omega \subseteq\left\{x \mid \lambda-l<x_{1}<\lambda\right\}$ be a bounded narrow region in $\Sigma_{\lambda}$ for $l>0$ small. Assume that $u \in \mathcal{L}_{\alpha} \cap C_{\text {loc }}^{1,1}$, $v \in \mathcal{L}_{\beta} \cap C_{\text {loc }}^{1,1}$ are lower semi-continuous on $\bar{\Omega}$. If $b_{i}(x)$ and $c_{i}(x)$ are positive and bounded from below in $\Omega, i=1,2$,

$$
\left\{\begin{array}{c}
(-\Delta)^{\frac{\alpha}{2}} U(x) \geq b_{1}(x) U(x)+c_{1}(x) V(x), \text { in } \Omega \\
(-\Delta)^{\frac{\beta}{2}} V(x) \geq b_{2}(x) U(x)+c_{2}(x) V(x), \text { in } \Omega \\
U(x) \geq 0, \quad V(x) \geq 0, \quad \text { in } \Sigma_{\lambda} \backslash \Omega \\
U_{\lambda}(x)=-U(x), \quad V_{\lambda}(x)=-V(x), \text { in } \Omega
\end{array}\right.
$$

then for sufficiently small $l$, we get

$$
U(x) \geq 0, \quad V(x) \geq 0, \quad \forall x \in \Omega .
$$

Furthermore, if $U(x)=0$ and $V(x)=0$ at some point in $\Omega$, then

$$
U(x)=0, \quad V(x)=0 \quad \text { a.e. } \quad x \in \mathbb{R}^{n} .
$$

For an unbounded narrow region $\Omega$, if we suppose

$$
\lim _{|x| \rightarrow \infty} U(x) \geq 0, \quad \lim _{|x| \rightarrow \infty} V(x) \geq 0,
$$

the above conclusions also hold.

Lemma 2.2 (Decay at Infinity) Let $\Omega$ be an unbounded region in $\Sigma_{\lambda}$. Assume that $u \in \mathcal{L}_{\alpha} \cap C_{l o c}^{1,1}, v \in \mathcal{L}_{\beta} \cap C_{l o c}^{1,1}$ satisfy the following equations

$$
\left\{\begin{array}{c}
(-\Delta)^{\frac{\alpha}{2}} U(x) \geq b_{1}(x) U(x)+c_{1}(x) V(x), \text { in } \Omega \\
(-\Delta)^{\frac{\beta}{2}} V(x) \geq b_{2}(x) U(x)+c_{2}(x) V(x), \text { in } \Omega \\
U(x) \geq 0, \quad V(x) \geq 0, \quad \text { in } \Sigma_{\lambda} \backslash \Omega \\
U_{\lambda}(x)=-U(x), \quad V_{\lambda}(x)=-V(x), \text { in } \Omega
\end{array}\right.
$$

where

$$
\begin{array}{lll}
b_{1}(x) \sim \frac{1}{|x|^{2 \alpha+\gamma}}, & c_{1}(x) \sim \frac{1}{|x|^{\alpha+\beta+\gamma}}, & |x| \rightarrow \infty, \\
b_{2}(x) \sim \frac{1}{|x|^{\alpha+\beta+\tau}}, & c_{1}(x) \sim \frac{1}{|x|^{2 \beta+\tau}}, & |x| \rightarrow \infty,
\end{array}
$$

and $b_{i}(x)$ and $c_{i}(x)$ are nonnegative in $\Omega, i=1,2$.

Then, there exists a constant $R$, which depends on $b_{i}(x)$ and $c_{i}(x), i=1,2$, but is independent of $U(x)$ and $V(x)$, such that if

$$
U\left(x^{0}\right)=\min _{x \in \Omega} U(x)<0, \quad V\left(x^{1}\right)=\min _{x \in \Omega} V(x)<0,
$$

then

$$
\left|x^{0}\right| \leq R, \quad \text { or } \quad\left|x^{1}\right| \leq R
$$


Proof. The proofs are analogous to the ones that [27]. For easily to read, we give outline of the proof.

By the elementary calculation, we derive

$$
\begin{aligned}
(-\Delta)^{\frac{\alpha}{2}} U\left(x^{0}\right) & =C_{n, \alpha} P V \int_{\mathbb{R}^{n}} \frac{U\left(x^{0}\right)-U(y)}{\left|x^{0}-y\right|^{n+\alpha}} d y \\
& =C_{n, \alpha} P V\left\{\int_{\Sigma_{\lambda}} \frac{U\left(x^{0}\right)-U(y)}{\left|x^{0}-y\right|^{n+\alpha}} d y+\int_{\mathbb{R}^{n} \backslash \Sigma_{\lambda}} \frac{U\left(x^{0}\right)-U(y)}{\left|x^{0}-y\right|^{n+\alpha}} d y\right\} \\
& =C_{n, \alpha} P V\left\{\int_{\Sigma_{\lambda}} \frac{U\left(x^{0}\right)-U(y)}{\left|x^{0}-y\right|^{n+\alpha}} d y+\int_{\Sigma_{\lambda}} \frac{U\left(x^{0}\right)-U_{\lambda}(y)}{\left|x^{0}-y^{\lambda}\right|^{n+\alpha}} d y\right\} \\
& =C_{n, \alpha} P V\left\{\int_{\Sigma_{\lambda}} \frac{U\left(x^{0}\right)-U(y)}{\left|x^{0}-y\right|^{n+\alpha}} d y+\int_{\Sigma_{\lambda}} \frac{U\left(x^{0}\right)+U(y)}{\left|x^{0}-y^{\lambda}\right|^{n+\alpha}} d y\right\} \\
& \leq C_{n, \alpha} P V \int_{\Sigma_{\lambda}} \frac{2 U\left(x^{0}\right)}{\left|x^{0}-y^{\lambda}\right|^{n+\alpha}} d y .
\end{aligned}
$$

For fixed $\lambda$, when $\left|x^{0}\right| \geq \lambda$ and $\left|x^{1}\right| \geq \lambda$, it is easy to derive

$$
\int_{\Sigma_{\lambda}} \frac{1}{\left|x^{0}-y^{\lambda}\right|^{n+\alpha}} d y \geq \frac{1}{\left|x^{0}\right|^{\alpha}} .
$$

Combining (2.14) with (2.15), we have

$$
(-\Delta)^{\frac{\alpha}{2}} U\left(x^{0}\right) \leq \frac{C}{\left|x^{0}\right|^{\alpha}} U\left(x^{0}\right) .
$$

Similarly, we obtain

$$
(-\Delta)^{\frac{\beta}{2}} V\left(x^{1}\right) \leq \frac{C}{\left|x^{1}\right|^{\beta}} V\left(x^{1}\right) .
$$

It follows from the first inequality of (2.10) and (2.16) that

$$
\frac{C}{\left|x^{0}\right|^{\alpha}} U\left(x^{0}\right) \geq c_{1}\left(x^{0}\right) V\left(x^{1}\right)
$$

Similarly, we obtain

$$
\frac{C}{\left|x^{1}\right|^{\beta}} V\left(x^{1}\right) \geq b_{2}\left(x^{1}\right) U\left(x^{1}\right) .
$$

From (2.18) and (2.19), we get

$$
\begin{aligned}
\frac{C}{\left|x^{0}\right|^{\alpha}} U\left(x^{0}\right) \geq c_{1}\left(x^{0}\right) V\left(x^{1}\right) & \geq c_{1}\left(x^{0}\right)\left|x^{1}\right|^{\beta} b_{2}\left(x^{1}\right) U\left(x^{1}\right) \\
& \geq c_{1}\left(x^{0}\right)\left|x^{1}\right|^{\beta} b_{2}\left(x^{1}\right) U\left(x^{0}\right) .
\end{aligned}
$$

That is,

$$
\frac{C}{\left|x^{0}\right|^{\alpha}\left|x^{1}\right|^{\beta} c_{1}\left(x^{0}\right) b_{2}\left(x^{1}\right)} \leq 1 .
$$

However, for $\left|x^{0}\right|$ and $\left|x^{1}\right|$ sufficiently large, the above inequality is equivalent to

$$
\left|x^{0}\right|^{\beta+\gamma}\left|x^{1}\right|^{\alpha+\tau},
$$


which is a contradiction.

Therefore, there exists $R>0$ such that

$$
\left|x^{0}\right| \leq R, \quad \text { or } \quad\left|x^{1}\right| \leq R .
$$

This completes the proof.

The following Lemma 2.3 and Lemma 2.4 are also crucical for us in [28].

Lemma 2.3. For $\lambda$ negative large, there exists a constant $C \geq 0$ and $\epsilon>0$ such that

$$
U_{\lambda}(x), V_{\lambda}(x) \geq C>0, x \in B_{\epsilon}\left(0^{\lambda}\right) \backslash\left\{0^{\lambda}\right\} .
$$

Lemma 2.4. For $\tilde{\lambda}<0$, if either of $U_{\tilde{\lambda}}(x), V_{\tilde{\lambda}}(x) \geq 0$, but not identically 0 . Then there exists a constant $C \geq 0$ and $\epsilon>0$ such that

$$
U_{\tilde{\lambda}}(x), V_{\tilde{\lambda}}(x) \geq C>0, x \in B_{\epsilon}\left(0^{\tilde{\lambda}}\right) \backslash\left\{0^{\tilde{\lambda}}\right\},
$$

where $\tilde{\lambda}$ defined as the following (3.12).

\section{Proof of Theorem 1.1 and Theorem 1.2}

\subsection{System in subcritical case}

In this subsection, we will use the method of moving planes to prove Theorem 1.1, namely, in the subcritical case, we show that (1.3) has no positive solution.

Proof of Theorem 1.1. By the definition of $U_{\lambda}$ and $V_{\lambda}$, we have

$$
\lim _{|x| \rightarrow \infty} U_{\lambda}(x)=0, \quad \lim _{|x| \rightarrow \infty} V_{\lambda}(x)=0 .
$$

Define

$$
\Sigma_{\lambda}^{u}=\left\{x \in \Sigma_{\lambda} \mid U_{\lambda}(x)<0\right\}, \quad \Sigma_{\lambda}^{v}=\left\{x \in \Sigma_{\lambda} \mid V_{\lambda}(x)<0\right\} .
$$

The proof consists of two steps.

Step 1. we show that when $\lambda$ sufficiently negative.

$$
U_{\lambda}(x), V_{\lambda}(x) \geq 0, \forall x \in \Sigma_{\lambda} \backslash\left\{0^{\lambda}\right\} .
$$

By an elementary calculation, for $x \in \Sigma_{\lambda}^{u} \cap \Sigma_{\lambda}^{v}$, we derive

$$
\begin{aligned}
(-\Delta)^{\frac{\alpha}{2}} U_{\lambda}(x)= & \frac{1}{\left|x^{\lambda}\right|^{a}} \bar{u}_{\lambda}^{p}(x) \bar{v}_{\lambda}^{q+1}(x)-\frac{1}{|x|^{a}} \bar{u}^{p}(x) \bar{v}^{q+1}(x) \\
= & \frac{1}{\left|x^{\lambda}\right|^{a}} \bar{u}_{\lambda}^{p}(x) \bar{v}_{\lambda}^{q+1}(x)-\frac{1}{|x|^{a}} \bar{u}_{\lambda}^{p}(x) \bar{v}_{\lambda}^{q+1}(x)-\frac{1}{|x|^{a}} \bar{u}_{\lambda}^{p}(x) \bar{v}_{\lambda}^{q+1}(x) \\
& -\frac{1}{|x|^{a}} \bar{u}^{p}(x) \bar{v}^{q+1}(x) \\
= & \left(\frac{1}{\left|x^{\lambda}\right| a}-\frac{1}{|x|^{a}}\right) \bar{u}_{\lambda}^{p}(x) \bar{v}_{\lambda}^{q+1}(x)+\frac{1}{|x|^{a}}\left(\bar{u}_{\lambda}^{p}(x) \bar{v}_{\lambda}^{q+1}(x)-\bar{u}^{p}(x) \bar{v}^{q+1}(x)\right) \\
\geq & \frac{1}{|x|^{a}}\left(\bar{u}_{\lambda}^{p}(x) \bar{v}_{\lambda}^{q+1}(x)-\bar{u}^{p}(x) \bar{v}^{q+1}(x)\right) \\
= & \frac{1}{|x|^{a}}\left(\bar{u}_{\lambda}^{p}(x) \bar{v}_{\lambda}^{q+1}(x)-\bar{u}_{\lambda}^{p}(x) \bar{v}^{q+1}(x)+\bar{u}_{\lambda}^{p}(x) \bar{v}^{q+1}(x)-\bar{u}^{p}(x) \bar{v}^{q+1}(x)\right) \\
\geq & \frac{1}{|x|^{a}}\left[\bar{u}_{\lambda}^{p}(x)\left(\bar{v}_{\lambda}^{q+1}(x)-\bar{v}^{q+1}(x)\right)+\left(\bar{u}_{\lambda}^{p}(x)-\bar{u}^{p}(x)\right) \bar{v}^{q+1}(x)\right] .
\end{aligned}
$$


For the above inequality, applying the Mean Value Theorem, we obtain

$$
\begin{aligned}
(-\Delta)^{\frac{\alpha}{2}} U_{\lambda}(x) & \geq \frac{C}{|x|^{a}}\left[\bar{u}_{\lambda}^{p}(x) \bar{v}^{q}(\xi)\left(\bar{v}_{\lambda}(x)-\bar{v}(x)\right)+\bar{v}^{q+1}(x) \bar{u}^{p-1}(\eta)\left(\bar{u}_{\lambda}(x)-\bar{u}(x)\right)\right] \\
& \geq \frac{C}{|x|^{a}} \bar{u}_{\lambda}^{p}(x) \bar{v}^{q}(x) V_{\lambda}(x)+\frac{C}{|x|^{a}} \bar{v}^{q+1}(x) \bar{u}^{p-1}(x) U_{\lambda}(x) \\
& \geq b_{1}(x) U_{\lambda}(x)+c_{1}(x) V_{\lambda}(x)
\end{aligned}
$$

where $\xi$ and $\eta$ are valued between $x^{\lambda}$ and $x$, and

$$
b_{1}(x)=\frac{C}{|x|^{a}} \bar{v}^{q+1}(x) \bar{u}^{p-1}(x), \quad c_{1}(x)=\frac{C}{|x|^{a}} \bar{u}_{\lambda}^{p}(x) \bar{v}^{q}(x) .
$$

Therefore, we obtain

$$
(-\Delta)^{\frac{\alpha}{2}} U_{\lambda}(x) \geq b_{1}(x) U_{\lambda}(x)+c_{1}(x) V_{\lambda}(x) .
$$

It is easy to derive that

$$
b_{1}(x) \sim \frac{1}{|x|^{2 \alpha+\gamma}}, \quad c_{1}(x) \sim \frac{1}{|x|^{\alpha+\beta+\gamma}}, \quad|x| \rightarrow \infty .
$$

Similarly, we get

$$
(-\Delta)^{\frac{\beta}{2}} V_{\lambda}(x) \geq b_{2}(x) U_{\lambda}(x)+c_{2}(x) V_{\lambda}(x),
$$

where

$$
b_{2}(x)=\frac{C}{|x|^{b}} \bar{u}^{p}(x) \bar{v}^{q}(x), \quad c_{2}(x)=\frac{C}{|x|^{b}} \bar{u}_{\lambda}^{p+1}(x) \bar{v}^{q-1}(x) .
$$

We have

$$
b_{2}(x) \sim \frac{1}{|x|^{\alpha+\beta+\tau}}, \quad c_{2}(x) \sim \frac{1}{|x|^{2 \beta+\tau}}, \quad|x| \rightarrow \infty .
$$

Suppose there exists some points $x^{0}$ such that

$$
U_{\lambda}\left(x^{0}\right)=\min _{x \in \Sigma_{\lambda}} U_{\lambda}(x)<0 .
$$

We claim that

$$
(-\Delta)^{\frac{\alpha}{2}} U_{\lambda}(x) \leq \frac{C}{\left|x^{0}\right|^{\alpha}} U_{\lambda}\left(x^{0}\right), \quad\left|x^{0}\right|>\lambda .
$$

In fact, we have

$$
\begin{aligned}
\int_{\Sigma_{\lambda}} \frac{1}{\left|x^{0}-y\right|^{n+\alpha}} d y & \geq \int_{B_{x^{0}\left(x^{1}\right)}} \frac{1}{\left|x^{0}-y\right|^{n+\alpha}} d y \\
& \geq \int_{B_{x^{0}\left(x^{1}\right)}} \frac{1}{4^{n+\alpha}\left|x^{0}\right|^{n+\alpha}} d y \\
& =\frac{\omega_{n}}{4^{n+\alpha}\left|x^{0}\right|^{\alpha}}
\end{aligned}
$$

where $\omega_{n}$ is the area of $n$ dimensional unit sphere and $B_{x^{0}}\left(x^{1}\right) \subset \mathbb{R}^{n} \backslash \Sigma_{\lambda}$ with $x^{1}=\left(3\left|x^{0}\right|+x_{1}^{0}, x_{2}, \cdots, x_{n}\right)$. 
By (2.14) and (3.5), we can derive that (3.4).

Combining (3.2) with (3.4), we obtain

$$
\frac{C}{\left|x^{0}\right|^{\alpha}} U_{\lambda}\left(x^{0}\right) \geq b_{1}\left(x^{0}\right) V_{\lambda}\left(x^{0}\right)+c_{1}\left(x^{0}\right) U_{\lambda}\left(x^{0}\right) .
$$

By the degeneracy of $b_{1}(x)$ at infinity and (3.6), for sufficiently negative $\lambda$,

$$
\frac{C}{\left|x^{0}\right|^{\alpha}} U_{\lambda}\left(x^{0}\right) \geq c_{1}\left(x^{0}\right) V_{\lambda}\left(x^{0}\right) .
$$

Now, we suppose that there is some point $x^{1}$ such that

$$
V_{\lambda}\left(x^{1}\right)=\min _{x \in \Sigma_{\lambda}} V_{\lambda}(x)<0 .
$$

Similar to (3.4), we obtain

$$
(-\Delta)^{\frac{\beta}{2}} V_{\lambda}(x) \leq \frac{C}{\left|x^{1}\right|^{\beta}} V_{\lambda}\left(x^{1}\right), \quad\left|x^{1}\right|>\lambda .
$$

Combining (3.3) with (3.8), we have

$$
\frac{C}{\left|x^{1}\right|^{\beta}} V_{\lambda}\left(x^{1}\right) \geq b_{2}\left(x^{1}\right) U_{\lambda}\left(x^{1}\right)+c_{2}\left(x^{1}\right) V_{\lambda}\left(x^{1}\right) .
$$

From the degeneracy of $c_{2}(x)$ at infinity and (3.9), for sufficiently negative $\lambda$, we have

$$
\frac{C}{\left|x^{1}\right|^{\beta}} V_{\lambda}\left(x^{1}\right) \geq b_{2}\left(x^{1}\right) U_{\lambda}\left(x^{1}\right) .
$$

Combining (3.7) with (3.10), we deduce

$$
\begin{aligned}
\frac{C}{\left|x^{1}\right|^{\beta}} V_{\lambda}\left(x^{1}\right) & \geq b_{2}\left(x^{1}\right) U_{\lambda}\left(x^{0}\right) \geq b_{2}\left(x^{1}\right)\left|x^{0}\right|{ }^{\alpha} c_{1}\left(x^{0}\right) V_{\lambda}\left(x^{0}\right) \\
& \geq b_{2}\left(x^{1}\right)\left|x^{0}\right|^{\alpha} c_{1}\left(x^{0}\right) V_{\lambda}\left(x^{1}\right) .
\end{aligned}
$$

Using the degeneracy of $b_{2}(x)$ and $c_{1}(x)$ at infinity, we have

$$
\frac{C}{\left|x^{1}\right|^{\beta}} V_{\lambda}\left(x^{1}\right) \geq \frac{1}{\left|x^{1}\right|^{\alpha+\beta+\tau}} \cdot \frac{1}{\left|x^{0}\right|^{\beta+\gamma}} V_{\lambda}\left(x^{1}\right) .
$$

Namely,

$$
\frac{1}{\left|x^{1}\right|^{\alpha+\tau}} \cdot \frac{1}{\left|x^{0}\right|^{\beta+\gamma}} \geq 1
$$

for sufficiently negative $\lambda$, the inequality does not hold.

From Lemma 2.2, for sufficiently negative $\lambda$, at least one of $U_{\lambda}$ and $V_{\lambda}$ are greater than or equal to 0 . Without loss of generality, we assume that

$$
U_{\lambda}(x) \geq 0, \quad x \in \Sigma_{\lambda} \backslash\left\{0^{\lambda}\right\} .
$$

The following proves that (3.11) to $V_{\lambda}$ is also true. In fact, if $V_{\lambda}$ is negative somewhere in $\Sigma_{\lambda} \backslash\left\{0^{\lambda}\right\}$, then there must exist some $\bar{x} \in \Sigma_{\lambda} \backslash\left\{0^{\lambda}\right\}$ such that

$$
V_{\lambda}(\bar{x})=\min _{x \in \Sigma_{\lambda}} V_{\lambda}(x)<0 .
$$


From previous arguments of (3.3) and (3.8), we have

$$
0>\frac{C}{|\bar{x}|^{\beta}} V_{\lambda}(\bar{x}) \geq(-\Delta)^{\frac{\beta}{2}} V_{\lambda}(\bar{x}) \geq b_{2}(\bar{x}) U_{\lambda}(\bar{x})+c_{2}(\bar{x}) V_{\lambda}(\bar{x})
$$

For the above inequality, combining with (3.10), we deduce

$$
\begin{aligned}
0 & >\frac{C}{|\bar{x}|^{\beta}} V_{\lambda}(\bar{x}) \\
& \geq b_{2}(\bar{x}) U_{\lambda}(\bar{x})+C b_{2}(\bar{x}) c_{2}(\bar{x})|\bar{x}|^{\beta} U_{\lambda}(\bar{x}) \\
& \geq 0 .
\end{aligned}
$$

It is a contradiction, and then we complete Step 1.

Step 2. The Step 1 provides a starting point, from which we can now move the plane $T_{\lambda}$ to the right as long as (3.1) holds to its limiting position. Let

$$
\tilde{\lambda}=\sup \left\{\lambda \leq 0 \mid U_{\rho} \geq 0, V_{\rho} \geq 0, \forall x \in \Sigma_{\rho} \backslash\left\{0^{\lambda}\right\}, \rho \leq \lambda\right\} .
$$

We claim that

$$
\tilde{\lambda}=0
$$

or

$$
U_{\tilde{\lambda}}(x) \equiv 0, V_{\tilde{\lambda}}(x) \equiv 0, \quad \forall x \in \Sigma_{\tilde{\lambda}} \backslash\left\{0^{\tilde{\lambda}}\right\} .
$$

If not, we suppose that $\tilde{\lambda}<0$, we have proved that the plane $T_{\lambda}$ can be moved further right. Namely, there exists some small $\delta>0$, such that for any $\lambda \in(\tilde{\lambda}, \tilde{\lambda}+\delta)$, we have

$$
U_{\lambda}(x) \geq 0, V_{\lambda}(x) \geq 0, \quad \forall x \in \Sigma_{\lambda} \backslash\left\{0^{\lambda}\right\},
$$

which is a contradiction with the definition of $\tilde{\lambda}$. Therefore, we deduce

$$
\tilde{\lambda}=0 .
$$

Practically, when $\tilde{\lambda}<0$, we have

$$
U_{\tilde{\lambda}}(x)>0, V_{\tilde{\lambda}}(x)>0, \quad \forall x \in \Sigma_{\tilde{\lambda}} \backslash\left\{0^{\tilde{\lambda}}\right\} .
$$

Otherwise, at least one of $U_{\tilde{\lambda}}(x)$ and $V_{\tilde{\lambda}}(x)$ is greater than or equal to zero.

Without loss of generality, we may assume that $U_{\lambda}(x) \geq 0$, namely, there exists some point $\tilde{x}$ such that

$$
U_{\tilde{\lambda}}(\tilde{x})=\min _{x \in \Sigma_{\tilde{\lambda}} \backslash\left\{0^{\tilde{\lambda}}\right\}} U_{\tilde{\lambda}}(x)=0 .
$$

It follows that

$$
\begin{aligned}
(-\Delta)^{\frac{\alpha}{2}} U_{\tilde{\lambda}}(\tilde{x}) & =C_{n, \alpha} P V \int_{\mathbb{R}^{n}} \frac{-U_{\tilde{\lambda}}(y)}{|\tilde{x}-y|^{n+\alpha}} d y \\
& =C_{n, \alpha} P V \int_{\Sigma_{\tilde{\lambda}}} \frac{-U_{\tilde{\lambda}}(y)}{|\tilde{x}-y|^{n+\alpha}} d y+C_{n, \alpha} P V \int_{\mathbb{R}^{n} \backslash \Sigma_{\tilde{\lambda}}} \frac{-U_{\tilde{\lambda}}(y)}{|\tilde{x}-y|^{n+\alpha}} d y \\
& =C_{n, \alpha} P V \int_{\Sigma_{\tilde{\lambda}}} \frac{-U_{\tilde{\lambda}}(y)}{|\tilde{x}-y|^{n+\alpha}} d y+C_{n, \alpha} P V \int_{\Sigma_{\tilde{\lambda}}} \frac{U_{\tilde{\lambda}}(y)}{\left|\tilde{x}-y^{\tilde{\lambda}}\right|^{n+\alpha}} d y \\
& \leq C_{n, \alpha} \int_{\Sigma_{\tilde{\lambda}}}\left(\frac{1}{\left|\tilde{x}-y^{\tilde{\lambda}}\right|^{n+\alpha}}-\frac{1}{|\tilde{x}-y|^{n+\alpha}}\right) U_{\tilde{\lambda}}(y) d y \\
& \leq 0 .
\end{aligned}
$$


On the other hand,

$$
\begin{aligned}
(-\Delta)^{\frac{\alpha}{2}} U_{\tilde{\lambda}}(\tilde{x})= & \frac{1}{\left|\tilde{x}^{\tilde{\lambda}}\right|^{a}} \bar{u}_{\tilde{\lambda}}^{p}(\tilde{x}) \bar{v}_{\tilde{\lambda}}^{q+1}(\tilde{x})-\frac{1}{|\tilde{x}|^{a}} \bar{u}^{p}(\tilde{x}) \bar{v}^{q+1}(\tilde{x}) \\
= & \frac{1}{\left|\tilde{x}^{\tilde{\lambda}}\right|^{a}} \bar{u}^{p}(\tilde{x}) \bar{v}_{\tilde{\lambda}}^{q+1}(\tilde{x})-\frac{1}{|\tilde{x}|^{a}} \bar{u}^{p}(\tilde{x}) \bar{v}^{q+1}(\tilde{x}) \\
= & \frac{1}{\left|\tilde{x}^{\tilde{\lambda}}\right|^{a}} \bar{u}^{p}(\tilde{x}) \bar{v}_{\tilde{\lambda}}^{q+1}(\tilde{x})-\frac{1}{|\tilde{x}|^{a}} \bar{u}^{p}(\tilde{x}) \bar{v}_{\tilde{\lambda}}^{q+1}(\tilde{x})+\frac{1}{|\tilde{x}|^{a}} \bar{u}^{p}(\tilde{x}) \bar{v}_{\tilde{\lambda}}^{q+1}(\tilde{x}) \\
& -\frac{1}{|\tilde{x}|^{a}} \bar{u}^{p}(\tilde{x}) \bar{v}^{q+1}(\tilde{x}) \\
= & \bar{u}^{p}(\tilde{x})\left[\left(\frac{1}{\mid \tilde{x}^{\tilde{\lambda} \mid a}}-\frac{1}{|\tilde{x}|^{a}}\right) \bar{v}_{\tilde{\lambda}}^{q+1}(\tilde{x})+\frac{1}{|\tilde{x}|^{a}}\left(\bar{v}_{\tilde{\lambda}}^{q+1}(\tilde{x})-\bar{v}^{q+1}(\tilde{x})\right)\right] \\
> & 0,
\end{aligned}
$$

which is a contradiction. Therefore, we obtain (3.15).

By Lemma 2.3 and Lemma 2.4, we claim that for $\tilde{\lambda}<0$ and $\epsilon>0$ sufficiently small,

$$
U_{\tilde{\lambda}}(x), V_{\tilde{\lambda}}(x) \geq C>0, \quad \forall x \in B_{\epsilon}\left(0^{\tilde{\lambda}}\right) \backslash\left\{0^{\tilde{\lambda}}\right\} .
$$

Combining with the above bounded away from 0 , we deduce that for $\delta>0$, there exists some constant $c_{0}>0$ such that

$$
U_{\tilde{\lambda}}(x), V_{\tilde{\lambda}}(x) \geq c_{0}, \quad \forall x \in\left(\Sigma_{\tilde{\lambda}-\delta} \backslash\left\{0^{\tilde{\lambda}}\right\}\right) \cap B_{R_{0}}(0) .
$$

For $\epsilon, \delta \ll|\tilde{\lambda}|, 0^{\tilde{\lambda}} \in\left(\Sigma_{\tilde{\lambda}-\delta} \backslash\left\{0^{\tilde{\lambda}}\right\}\right) \cap B_{R_{0}}(0)$, since $U_{\lambda}$ and $V_{\lambda}$ depend on $\lambda$ continuously, we have

$$
U_{\tilde{\lambda}}(x), V_{\tilde{\lambda}}(x) \geq 0, \quad \forall x \in\left(\Sigma_{\tilde{\lambda}-\delta} \backslash\left\{0^{\tilde{\lambda}}\right\}\right) \cap B_{R_{0}}(0) .
$$

By Lemma 2.2, we know that if

$$
U_{\lambda}(\hat{x})=\min _{\Sigma_{\lambda}} U_{\lambda}<0
$$

then there exists a large $R_{0}$ such that

$$
|\hat{x}| \leq R_{0}
$$

Hence $\hat{x} \in\left(\Sigma_{\lambda} \backslash \Sigma_{\lambda-\delta}\right) \cap B_{R_{0}}(0)$.

For sufficiently large $R_{0}$, similar to (3.4), we obtain

$$
V_{\lambda}(\hat{x})<0 .
$$

Therefore, there exists some point $\bar{x}$ such that

$$
V_{\lambda}(\bar{x})=\min _{\Sigma_{\lambda}} V_{\lambda}<0 .
$$

If $\bar{x} \in B_{R_{0}}^{C} \cap \Sigma_{\lambda}$, similar to (3.10), we have

$$
0>\frac{C}{|\bar{x}|^{\beta}} V_{\lambda}(\bar{x}) \geq b_{2}(\bar{x}) U_{\lambda}(\bar{x}) .
$$


Meanwhile, for $U_{\lambda}$ at $\hat{x}$, similar to (3.7), we have

$$
\frac{C}{|\epsilon+\delta|^{\alpha}} U_{\lambda}(\hat{x}) \geq c_{1}(\hat{x}) V_{\lambda}(\hat{x})
$$

By (3.18) and (3.19), we have

$$
b_{2}(\bar{x})|\bar{x}|^{\beta} c_{1}(\hat{x})|\epsilon+\delta|^{\alpha} \geq C .
$$

We know that $c_{1}(\hat{x})$ is bounded, and $b_{2}(\bar{x})|\bar{x}|^{\beta}$ is also bounded for $|\bar{x}|>R_{0}$. Hence for $\epsilon, \delta$ sufficiently small, (3.20) does not hold namely, $\bar{x} \notin B_{R_{0}}^{C} \cap \Sigma_{\lambda}$.

Combining with Lemma 2.1, let

$$
\text { narrow region } \Omega=\left(\Sigma_{\lambda} \backslash \Sigma_{\lambda-\delta}\right) \cap B_{R_{0}}(0),
$$

while $U_{\lambda}$ and $V_{\lambda}$ satisfy system (2.8), we have

$$
U_{\lambda}(x), V_{\lambda}(x) \geq 0, \quad \forall x \in\left(\Sigma_{\lambda} \backslash \Sigma_{\lambda-\delta}\right) \cap B_{R_{0}}(0) .
$$

Now, we conclude that neither $U_{\lambda}$ nor $V_{\lambda}$ has negative minimum in $\Sigma_{\lambda} \backslash\left\{0^{\lambda}\right\}$. Therefore, we obtain

$$
U_{\lambda}(x), V_{\lambda}(x) \geq 0, \quad \forall x \in \Sigma_{\lambda} \backslash\left\{0^{\lambda}\right\} .
$$

This completes the proof of (3.15). Therefore we have

$$
\tilde{\lambda}=0 .
$$

Similarly, we can move the plane from $x_{1}=+\infty$ near to the left, and we can show that

$$
U_{\lambda}(x), V_{\lambda}(x) \leq 0, \quad \forall x \in \Sigma_{\lambda} \backslash\left\{0^{\lambda}\right\} .
$$

Therefore, we deduce

$$
\tilde{\lambda}=0, U_{\tilde{\lambda}}(x) \equiv 0, V_{\tilde{\lambda}}(x) \equiv 0, \quad \forall x \in \Sigma_{\tilde{\lambda}} \backslash\left\{0^{\tilde{\lambda}}\right\} .
$$

Since the direction of $x_{1}$-axis is arbitrary, we have $\bar{u}$ and $\bar{v}$ are radially symmetric about the origin.

For any point $z^{0} \in \mathbb{R}^{n}$ apply the Kelvin transform centered at $z^{0}$, and by an entirely similar argument, one can show that $\bar{u}$ and $\bar{v}$ are radially symmetric about $z^{0}$.

Let $z^{1}$ and $z^{2}$ be any two points in $\mathbb{R}^{n}$ and we choose the coordinate system so that the midpoint

$$
z^{0}=\frac{z^{1}+z^{2}}{2}
$$

is the origin. Since $\bar{u}$ and $\bar{v}$ are radially symmetric about $z^{0}$, we have

$$
u\left(z^{1}\right)=u\left(z^{2}\right), \quad v\left(z^{1}\right)=v\left(z^{2}\right) .
$$

This implies that $u$ and $v$ must be constants.

But positive constant solutions do not satisfy system (1.3). Namely, in subcritical case, there is no positive solution for system (1.3). 


\subsection{System in critical case}

In this subsection, we still utilize the Kelvin transform of $u$ and $v$ centered at the origin. In critical case, namely, $n+\alpha+\gamma-p(n-\alpha)-(q+1)(n-\beta)=$ $0, n+\alpha+\gamma-(p+1)(n-\alpha)-q(n-\beta)=0$, we will show that either $\bar{u}$ and $\bar{v}$ are symmetric about the origin or some point.

We still use the notations in the subcritical case. The argument is quite similar to, but not entirely the same as that in the subcritical case. Hence we still present some details here.

Proof of Theorem 1.2. In critical case, similar to (3.2), we have

$$
(-\Delta)^{\frac{\alpha}{2}} U_{\lambda}(x) \geq \bar{b}_{1}(x) U_{\lambda}(x)+\bar{c}_{1}(x) V_{\lambda}(x),
$$

where $\bar{b}_{1}(x)=C \bar{u}^{p-1}(x) \bar{v}^{q+1}(x), \bar{c}_{1}(x)=C \bar{u}_{\lambda}^{p}(x) \bar{v}^{q}(x)$.

Similarly, we get

$$
(-\Delta)^{\frac{\beta}{2}} V_{\lambda}(x) \geq \bar{b}_{2}(x) U_{\lambda}(x)+\bar{c}_{2}(x) V_{\lambda}(x),
$$

where $\bar{b}_{2}(x)=C \bar{u}^{p}(x) \bar{v}^{q}(x), \bar{c}_{2}(x)=C \bar{u}_{\lambda}^{p+1}(x) \bar{v}^{q-1}(x)$.

It is easy to derive that

$$
\begin{aligned}
& \bar{b}_{1}(x) \sim \frac{1}{|x|^{2 \alpha+\gamma}}, \quad \bar{c}_{1}(x) \sim \frac{1}{|x|^{\alpha+\beta+\gamma}}, \quad \text { as }|x| \rightarrow \infty . \\
& \bar{b}_{2}(x) \sim \frac{1}{|x|^{\alpha+\beta+\gamma}}, \quad \bar{c}_{2}(x) \sim \frac{1}{|x|^{2 \beta+\gamma}}, \quad \text { as }|x| \rightarrow \infty .
\end{aligned}
$$

The remaining proof is the same as that in the subcritical case.

We can show that, for $\lambda$ sufficiently negative,

$$
U_{\lambda}(x), V_{\lambda}(x) \geq 0, \quad \forall x \in \Sigma_{\lambda} \backslash\left\{0^{\lambda}\right\} .
$$

Defined

$$
\tilde{\lambda}=\sup \left\{\lambda \leq 0 \mid U_{\rho} \geq 0, V_{\rho} \geq 0, \forall x \in \Sigma_{\rho} \backslash\left\{0^{\lambda}\right\}, \rho \leq \lambda\right\} .
$$

We consider two possible cases.

Case 1. $\tilde{\lambda}<0$

For this case, either

$$
U_{\tilde{\lambda}}(x)=V_{\tilde{\lambda}}(x) \equiv 0, x \in \Sigma_{\tilde{\lambda}} \backslash\left\{0^{\tilde{\lambda}}\right\}
$$

or

$$
U_{\tilde{\lambda}}(x), V_{\tilde{\lambda}}(x)>0, x \in \Sigma_{\tilde{\lambda}} \backslash\left\{0^{\tilde{\lambda}}\right\} .
$$

We suppose that there exists some point $\tilde{x} \in \Sigma_{\tilde{\lambda}}$ such that

$$
U_{\tilde{\lambda}}(\tilde{x})=\min _{x \in \Sigma_{\tilde{\lambda}}} U_{\tilde{\lambda}}(x)=0,
$$

then

$$
U_{\tilde{\lambda}}(x) \equiv 0, x \in \Sigma_{\tilde{\lambda}} \backslash\left\{0^{\tilde{\lambda}}\right\} .
$$

Otherwise,

$$
(-\Delta)^{\frac{\alpha}{2}} U_{\tilde{\lambda}}(\tilde{x})=C_{n, \alpha} P V \int_{\mathbb{R}^{n}} \frac{-U_{\tilde{\lambda}}(y)}{|\tilde{x}-y|^{n+\alpha}} d y<0 .
$$


On the other hand,

$$
\begin{aligned}
(-\Delta)^{\frac{\alpha}{2}} U_{\tilde{\lambda}}(\tilde{x}) & =\bar{u}_{\tilde{\lambda}}^{p}(\tilde{x}) \bar{v}_{\tilde{\lambda}}^{q+1}(\tilde{x})-\bar{u}^{p}(\tilde{x}) \bar{v}^{q+1}(\tilde{x}) \\
& =\bar{u}^{p}(\tilde{x})\left(\bar{v}_{\tilde{\lambda}}^{q+1}(\tilde{x})-\bar{v}^{q+1}(\tilde{x})\right) \\
& \geq 0 .
\end{aligned}
$$

It is a contradition. Hence (3.22) holds.

When $U_{\tilde{\lambda}}(x) \equiv 0$, by the anti-symmetry of $U_{\lambda}$, namely,

$$
U_{\tilde{\lambda}}(x)=-U_{\tilde{\lambda}}\left(x^{\tilde{\lambda}}\right) .
$$

We derive that

$$
U_{\tilde{\lambda}}(x) \equiv 0, \quad x \in \mathbb{R}^{n}
$$

Therefore, we have

$$
(-\Delta)^{\frac{\alpha}{2}} U_{\tilde{\lambda}}(x)=0 .
$$

Since

$$
\begin{aligned}
(-\Delta)^{\frac{\alpha}{2}} U_{\tilde{\lambda}}(\tilde{x}) & =\bar{u}_{\tilde{\lambda}}^{p}(\tilde{x}) \bar{v}_{\tilde{\lambda}}^{q+1}(\tilde{x})-\bar{u}^{p}(\tilde{x}) \bar{v}^{q+1}(\tilde{x}) \\
& =\bar{u}^{p}(\tilde{x})\left(\bar{v}_{\tilde{\lambda}}^{q+1}(\tilde{x})-\bar{v}^{q+1}(\tilde{x})\right) \\
& =0 .
\end{aligned}
$$

It must be true that

$$
\bar{v}_{\tilde{\lambda}}^{q+1}(\tilde{x})=\bar{v}^{q+1}(\tilde{x}), \quad x \in \mathbb{R}^{n} .
$$

Therefore, we have

$$
V_{\tilde{\lambda}}(x) \equiv 0, \quad x \in \mathbb{R}^{n} .
$$

Similarly, if $V_{\tilde{\lambda}}(x)=0$ somewhere, then we can prove that

$$
U_{\tilde{\lambda}}(x) \equiv 0, \quad x \in \mathbb{R}^{n} .
$$

When (3.21) holds, by using an entirely similar argument of Step 2 in subcritical case. One can keep moving the plane $T_{\lambda}$, namely, there exists some small $\delta>0$, such that for any $\lambda \in(\tilde{\lambda}, \tilde{\lambda}+\delta)$, we have

$$
U_{\lambda}(x) \geq 0, V_{\lambda}(x) \geq 0, \quad \forall x \in \Sigma_{\lambda} \backslash\left\{0^{\lambda}\right\},
$$

which is a contradiction with the definition of $\tilde{\lambda}$. Therefore (3.21) must not be true.

We conclude that

$$
U_{\tilde{\lambda}}(x)=V_{\tilde{\lambda}}(x) \equiv 0, x \in \mathbb{R}^{n} .
$$

This implies $u$ and $v$ are symmetric about some point in $\mathbb{R}^{n}$.

Case 2. $\tilde{\lambda}=0$ that

In this case, we can move the plane from near $x_{1}=+\infty$ to the left, and derive

$$
U_{0}(x), V_{0}(x)>0, \quad \forall x \in \Sigma_{0} .
$$

Hence, $U_{0}(x), V_{0}(x) \equiv 0, \forall x \in \Sigma_{0}$.

This proves that $\bar{u}$ and $\bar{v}$ are symmetric about the origin. So are $u$ and $v$. 


\section{Proof Theorem 1.4}

Proof. We claim that if (1.3) does not admit any bounded solutions in $\mathbb{R}^{n}$. Then there exists $C=C(n, p, q)>0$ such that any solutions $(u, v)$ of (1.16) satisfies (1.17) and (1.18). If not, there exists sequences $\Omega_{k},\left(u_{k}, v_{k}\right), y_{k} \in \Omega_{k}$ such that $\left(u_{k}, v_{k}\right)$ solves (1.3) on $\Omega_{k}$ and

$$
M_{k}:=u_{k}^{\frac{p+q}{\alpha}}+v_{k}^{\frac{p+q}{\beta}}, k=1,2, \cdots
$$

satisfies $M\left(y_{k}\right)>2 k$ dist $^{-1}\left(y_{k}, \Omega_{k}\right)$. By the Doubling Lemma, it follows that there exist $x_{k} \in \Omega_{k}$ such that

$$
\begin{gathered}
M_{k}\left(x_{k}\right)>2 k \operatorname{dist}^{-1}\left(x, \Omega_{k}\right), \\
M_{k}(z) \leq 2 M_{k}\left(x_{k}\right),\left|z-x_{k}\right| \leq k M_{k}^{-1}\left(x_{k}\right) .
\end{gathered}
$$

Write $\rho_{k}=M_{k}^{-1}\left(x_{k}\right)$ and

$$
\tilde{u}_{k}(y):=\rho_{k}^{\frac{\alpha}{p+q}} u_{k}\left(x_{k}+\rho_{k} y\right), \tilde{v}_{k}(y):=\rho_{k}^{\frac{\beta}{p+q}} v_{k}\left(x_{k}+\rho_{k} y\right),|y| \leq k .
$$

Clearly, $\tilde{u}_{k}, \tilde{v}_{k}$ are also solutions of system (1.3) for $|y| \leq k$, and they also satisfy

$$
\begin{gathered}
{\left[\tilde{u}_{k}^{\frac{p+q}{\alpha}}+\tilde{v}_{k}^{\frac{p+q}{\beta}}\right](0)=1,} \\
{\left[\tilde{u}_{k}^{\frac{p+q}{\alpha}}+\tilde{v}_{k}^{\frac{p+q}{\beta}}\right](y) \leq 2,|y| \leq k .}
\end{gathered}
$$

By using Theorem 1.3 in [11] and Lemma 2.9 in [21], we deduce that some subsequence of $\left(\tilde{u}_{k}, \tilde{v}_{k}\right)$ converges in $C_{l o c}^{\varepsilon+\mu}$ to solutions $(\tilde{u}, \tilde{v})$ of $(1.3)$ in $\mathbb{R}^{n}$, where $\varepsilon$ and $\mu$ satisfies the following conditions: for any $\varepsilon>0$ and $\mu \in(0,2)$, such that $\varepsilon \notin \mathbb{N}$, and $\varepsilon+\mu \notin \mathbb{N}$. It a contradictions with the assumption of Theorem 1.1.

On the contrary, if the positive solution $(u, v)$ of (1.3) satisfies the estimate (1.17) and (1.18), by letting $\Omega \rightarrow \mathbb{R}^{n}$, we deduce

$$
u=v=0, \quad \text { in } \mathbb{R}^{n} .
$$

This shows the nonexistence of positive solution.

Acknowledgements. This project is supported by National Natural Science Foundation of China (Grant No. 11571093).

Data Availability Statement: All data generated or analysed during this study are included in this published article (and its supplementary information files).

\section{References}

[1] Barlow M, Bass, R, Chen Z, Kassmann M, Non-local Dirichlet forms and symmetric jump processes. Trans. Amer. Math. Soc. 361(2009):1963-1999.

[2] Bidaut-VronMarie-Francoise, RaouxThierry, Asymptotics of solutions of some nonlinear elliptic systems. Communications in Partial Differential Equations, 21(1996):1035-1086. 
[3] Bouchaud J, Georges A, Anomalous diffusion in disordered media: Statistical mechanisms, models and physical applications. Physics Reports, 195(1990):127-293.

[4] Caffarelli L, Silvestre L, An extension problem related to the fractional Laplacian, Comm. Partial Differential Equations 32(2007):1245-1260.

[5] Caffarelli L, Vasseur L, Drift diffusion equations with fractional diffusion and the quasi-geostrophic equation, Ann. of Math. 171(2010):1903-1930.

[6] Chen W, Li C, Super polyharmonic property of solutions for PDE systems and its applications, Commun. Pure Appl. Anal., 12(2013):2497-2514.

[7] Chen W, Li C, Li Y, A direct method of moving planes for the fractional Laplacian. Advances in Mathematics, 308(2017):404-437.

[8] David A, Lévy Processes-From Probability to Finance and Quantum Groups, Notices of the American Mathematical Society (American Mathematical Society), 2014:13361347 .

[9] Chen W, Fang Y, Yang R, Liouville theorems involving the fractional Laplacian on a half space. Advances in Mathematics, 274(2015):167-198.

[10] Duong A, Le P. Symmetry and nonexistence results for a fractional Hnon-Hardy system on a half-space. Rocky Mountain Journal of Mathematics, 49(2019):789-816.

[11] Dipierro S, Savin O, Valdinoci E, Definition of fractional Laplacian for functions with polynomial growth. Revista Matematica Iberoamericana, 35(2019): 1079-1122.

[12] Li D, Niu P, Zhuo R, Symmetry and nonexistence of positive solutions of integral systems with Hardy terms. Journal of Mathematical Analysis Applications, 424(2015):915-931.

[13] Li Y, Liu B, Singularity estimates for elliptic systems of m-laplacians. Journal of the Korean Mathematical Society, 55(2018):1423-1433.

[14] Li Y, Lei Y, On existence and nonexistence of positive solutions of an elliptic system with coupled terms. Communications on Pure and Applied Analysis, 17(2018):17491764 .

[15] Lei Y, On the regularity of positive solutions of a class of Choquard type equations. Mathematische Zeitschrift, 273(2013): 883-905.

[16] Lei Y, Li C, Ma C, Asymptotic radial symmetry and growth estimates of positive solutions to weighted Hardy-Littlewood-Sobolev system of integral equations, Calc. Var. Partial Differential Equations, 45(2012):43-61.

[17] Li C, Ma L, Uniqueness of positive bound states to Schrödinger systems with critical exponents, SIAM J. Math. Anal. 40(2008):1049-1057.

[18] Ma P, Li Q, Li Y, A Pohozaev Identity for the Fractional Hénon System. Acta Mathematica Sinica, English Series, 33(2017):1382-1396.

[19] Ma P, Li Y, Zhang J. Symmetry and nonexistence of positive solutions for fractional systems. 17(2018):1053-1070. 
[20] Peter P, Quittner P, Souplet P, Singularity and decay estimates in superlinear problems via Liouville-type theorems, I: Elliptic equations and systems. Duke Mathematical Journal, 139(2007):555-579.

[21] Ros-Oton X, Serra J, The Dirichlet problem for the fractional Laplacian: Regularity up to the boundary. Journal De Mathematiques Pures Et Appliquées, 101(2014):275302.

[22] Ros-Oton X, Serra J, Fractional Laplacian: Pohozaev identity and nonexistence results, C. R. Math. Acad. Sci. Paris, 350(2012):505-508.

[23] Ros-Oton X, Serra J, The Pohozaev Identity for the Fractional Laplacian. Arch. Rational Mechan. Anal., 213(2014):587-628.

[24] Tarasov V, Zaslavsky G, Fractional dynamics of systems with long-range interaction. Communications in Nonlinear Science Numerical Simulation, 11(2006):885-898.

[25] Zaslavsky G, Hamiltonian Chaos and Fractional Dynamics. Oxford University Press, England (2008).

[26] Zhuo R, Li Y, A Liouville theorem for the higher-order fractional Laplacian. Communications in Contemporary Mathematics, 21(2019):https://doi.org/10.1142/S0219199718500050.

[27] Zhuo R, Li Y, Nonexistence and symmetry of solutions for schrodinger system$\mathrm{s}$ involving fractional Laplacian. Discrete and Continuous Dynamical Systems, 39(2019):1595-1611.

[28] Zhang R, Wang X, Yang Z, Symmetry and Nonexistence of Positive Solutions for an Elliptic System Involving the Fractional Laplacian. Quaestiones Mathematicae, (2021), 1-19. 